\title{
Androgen signalling and steroid receptor crosstalk in endocrine cancers
}

\author{
Frank Claessens and Wayne Tilley ${ }^{1}$ \\ Molecular Endocrinology Laboratory, Department of Cellular and Molecular Medicine, KU Leuven, Herestraat 49, \\ Campus GHB ON1, PO Box 901, 3000 Leuven, Belgium \\ 'Dame Roma Mitchell Cancer Research Laboratories, School of Medicine, University of Adelaide, Adelaide, \\ South Australia 5000, Australia
}

Correspondence should be addressed to F Claessens Email

Frank.claessens@ med.kuleuven.be

\section{Introduction}

In this issue, we bring together some fascinating aspects and novel insights into androgen signalling in endocrine cancers, focusing mostly on prostate cancer, but inclusive of breast, ovarian and endometrial cancers. One intriguing area of investigation covered herein is the crosstalk between androgen receptor (AR) and estrogen receptor (ER) signalling, not only in breast cancer but also in prostate cancer. Although it has long been known that androgen and estrogen sex hormones exert antagonistic influences during sexual development, the significance of their interplay and its consequences for disease development and progression are only beginning to be explored at the molecular level. Certainly, the intertwining of these two distinct research domains will provide fertile ground for new discoveries and will feature strongly in future endocrine-related cancer research.

\section{Androgens and prostate cancer}

Androgens are a major driver of prostate development, through the activation of different gene expression programs in stromal and epithelial cells and via modulation of the interactions between malignant and nonmalignant prostate epithelial cells. Even in the metastatic, castration-resistant forms of prostate cancer, androgens are key survival factors. Thanks to an integration of data on whole genome AR-binding sites and androgen-responsive gene networks, researchers are now starting to unravel how androgens and the AR mechanistically regulate cell survival, proliferation and cancer-specific changes in metabolism in prostate cancer cells. Barfeld et al. (2014) bring clarity to how androgens impact on the different hallmarks of cancer of the prostate.

Several older and newer therapies for metastatic prostate cancer target the AR signalling axis. Unfortunately, prostate cancer cells seem able to readily adapt to these therapies via a variety of mechanisms. Under chemical castration conditions, autonomous androgen synthesis can occur in cancer cells, whereby expression of enzymes involved in androgen synthesis is enhanced and expression of enzymes involved in androgen metabolism is diminished. This altered intracrinology is now being targeted clinically by use of CYP17A1 inhibitors. Penning discusses the different enzymes involved in intracrine androgen homoeostasis and the dangers and pitfalls that need to be taken into account in the further development of compounds that adopt this type of therapeutic strategy (Penning 2014).

Several other mechanisms have been described that might explain the maintenance of AR signalling under conditions of low androgen levels in castrate-resistant prostate cancer which involve changes in levels or intracellular location of AR-associated co-regulatory proteins (Gonugunta et al. 2014) or AR-regulated miRNAs (Selth et al. 2012). Gonugunta et al. (2014) describe the role of PELP1, not only in the regulation of AR activity in prostate cancer, but also in the regulation of ER activity in breast cancer. Another mechanism that explains the continued role of AR as a survival factor for prostate cancer cells, even under hormone deprivation, is the expression 
of truncated AR variants that are no longer dependent on hormone for activation. From Ware et al. (2014), we learn that these variants are clinically relevant prognostic factors in prostate cancer, for which the development of new therapies and markers are desperately needed.

It is becoming increasingly clear that the AR remains an important drug target in metastatic prostate cancer, even in castration-resistant forms of the disease, including resistance to enzalutamide and/or or abiraterone. This warrants the continued search for alternative AR antagonists, which target either the ligand-binding domain or new strategies that target the amino-terminal domain of the AR (reviewed in Helsen et al. (2014)).

It is extremely important to realise that there is a downside of systemic AR targeting in prostate cancer. Indeed, as summarised in this issue by Allan et al. (2014), complications of androgen deprivation should be closely monitored and managed clinically, and preclinical work should endeavour to prevent these side effects which negatively impact on quality of life, e.g. by developing selective AR antagonists.

Finally, the AR, like most steroid receptors has long been known to be a substrate for kinases and phosphatases. While the specific functions of ER phosphorylation have been described in great detail (Skliris et al. 2010), the reports on AR post-translational modifications have been confusing. Koryakina et al. (2014) start to bring clarity to this field, reviewing the current status of the kinases and phosphatases that target the AR and their possible role in prostate cancer.

\section{Androgens in breast and other female tissues}

Nieto et al. (2014) summarise the growing evidence on the significance of AR signalling in breast development and homoeostasis in female mice, while McNamara et al. (2014) and Tarulli et al. (2014) focus on the emerging role of the AR in breast cancer. It has been known for decades that AR is expressed in a large proportion of breast cancers and that clinical and laboratory evidence indicated that AR signalling regulates the activity of breast cancer cells. However, the complexity of this activity and its apparent plasticity depending on disease context has only recently been appreciated. McNamara et al. (2014) summarise recent data on the complexities of AR signalling in breast cancer, including the clinical utility of assessing serum androgen levels or AR expression levels as prognostic factors in breast cancer, how ER status influences AR action in breast cancer cells and the role of AR/ER molecular crosstalk in this disease. This work is highly topical as clinical trials examining the efficacy of AR-targeting therapies (used for prostate cancer) in women with metastatic breast cancer are currently underway. On a more hypothesis-generating angle, Tarulli et al. (2014) integrate recent data on the role of AR and NOTCH signalling in breast development to propose that crosstalk between these pathways plays a significant role in breast morphogenesis and carcinogenesis.

Extending beyond the breast, Gibson et al. (2014) discuss the AR, not only as a possible factor in the aetiology of endometrial and ovarian cancers, but also its potential use as a therapeutic target for these diseases. In endometrial cancer, androgens seem to have an antiproliferative effect, while in ovarian cancers androgens seem to have pro-proliferative effect. This article highlights a fertile new area for future research and is also an example of how investigation of AR signalling in endocrine-sensitive female tissues has largely been neglected.

\section{Estrogens in prostate cancer}

Testosterone activates the AR, but can also be converted into estrogen by aromatization in both females and males. Estrogens are the natural ligand for two receptors: ER $\alpha$ (ESR1) and ER $\beta$ (ESR2). Surprisingly, these two receptors have very different roles, not only in biology but also in pathologies such as breast and prostate cancers. Nelson et al. (2014) discuss the emerging roles of the two ERs and variants thereof in prostate cancer biology. This article complements the one by Gonugunta et al. (2014), which reviews evidence for crosstalk between AR and ER $\beta$ via the PELP protein in prostate cancer. The article by Nelson et al. (2014) is very topical and like the articles examining AR activity in female endocrine cancers highlights an avenue for future research in male endocrinopathies that has been relatively ignored.

\section{Conclusions}

Clearly, basic knowledge on sex steroid signalling in prostate and breast cancers is rapidly progressing, with cross fertilisation taking place because of the emerging roles of ERs in prostate cancer and AR in female cancers of the breast, ovary and endometrium. While many basic molecular aspects need further elucidation, new therapeutic targets and clinical applications are already apparent. It is only through collaborations and exchange of ideas that we will be able to step toward the goal of personalised medical approaches to the treatment and

Published by Bioscientifica Ltd. 
prevention of breast and prostate cancer, and extend these to encompass other endocrine cancers. We hope that this series of articles inspires further cross fertilisation of thought and investigative effort to achieve these important goals.

\section{Declaration of interest}

The authors declare that there is no conflict of interest that could be perceived as prejudicing the impartiality of this editorial.

\section{Funding}

This editorial did not receive any specific grant from any funding agency in the public, commercial or not-for-profit sector.

\section{References}

Allan CA, Collins VR, Frydenberg M, McLachlan RI \& Matthiesson KL 2014 Androgen deprivation therapy complications. Endocrine-Related Cancer 21 T119-T129. (doi:10.1530/ERC-13-0467)

Barfeld SJ, Itkonen HM, Urbanucci A \& Mills IG 2014 Androgen-regulated metabolism and biosynthesis in prostate cancer. Endocrine-Related Cancer 21 T57-T66. (doi:10.1530/ERC-13-0515)

Gibson DA, Simitsidellis I, Collins F \& Saunders PT 2014 Evidence of androgen action in endometrial and ovarian cancers. Endocrine-Related Cancer 21 T203-T218. (doi:10.1530/ERC-13-0551)

Gonugunta VK, Miao L, Sareddy GR, Ravindranathan P, Vadlamudi R \& Raj GV 2014 The social network of PELP1 and its implications in breast and prostate cancers. Endocrine-Related Cancer 21 T79-T86. (doi:10.1530/ERC-13-0502)

Helsen C, Van den Broeck T, Voet A, Prekovic S, Van Poppel H, Joniau S \& Claessens F 2014 Androgen receptor antagonists for prostate cancer therapy. Endocrine-Related Cancer 21 T105-T118. (doi:10.1530/ ERC-13-0545)

Koryakina Y, Ta HQ \& Gioeli D 2014 Androgen receptor phosphorylation: biological context and functional consequences. Endocrine-Related Cancer 21 T131-T145. (doi:10.1530/ERC-13-0472)

McNamara K, Moore N, Hickey T, Sasano H \& Tilley W 2014 Complexities of androgen receptor signalling in breast cancer. Endocrine-Related Cancer 21 T161-T181. (doi:10.1530/ERC-14-0243)

Nelson AW, Tilley WD, Neal DE \& Carroll JS 2014 Oestrogen receptor $\beta$ in prostate cancer: friend or foe? Endocrine-Related Cancer 21 T219-T234. (doi:10.1530/ERC-13-0508)

Nieto CM, Rider LC \& Cramer SD 2014 Influence of stromal-epithelial interactions on androgen action. Endocrine-Related Cancer 21 T147-T159. (doi:10.1530/ERC-14-0138)

Penning TM 2014 Androgen biosynthesis in castration resistant prostate cancer. Endocrine-Related Cancer 21 T67-T78. (doi:10.1530/ERC-14-0109)

Selth LA, Tilley WD \& Butler LM 2012 Circulating microRNAs: macroutility as markers of prostate cancer? Endocrine-Related Cancer 19 R99-R113. (doi:10.1530/ERC-12-0010)

Skliris GP, Nugent ZJ, Rowan BG, Penner CR, Watson PH \& Murphy LC 2010 A phosphorylation code for oestrogen receptor- $\alpha$ predicts clinical outcome to endocrine therapy in breast cancer. Endocrine-Related Cancer 17 589-597. (doi:10.1677/ERC-10-0030)

Tarulli G, Butler L, Tilley W \& Hickey T 2014 Moving androgens up a notch in breast cancer. Endocrine-Related Cancer 21 T183-T202. (doi:10.1530/ ERC-14-0248)

Ware KE, Garcia-Blanco MA, Armstrong AJ \& Dehm SM 2014 Biologic and clinical significance of androgen receptor variants in castration resistant prostate cancer. Endocrine-Related Cancer 21 T87-T103. (doi:10.1677/ERC-13-0470)

Received in final form 5 June 2014

Accepted 6 June 2014

Made available online as an Accepted Preprint

9 June 2014
(C) 2014 Society for Endocrinology Printed in Great Britain
Published by Bioscientifica Ltd. 